\title{
Role of the Finger Flexors in Rheumatoid Deformities of the Metacarpophalangeal Joints
}

\author{
By Edwin M. Smith, Robert C. Juvinall, Leonard F. Bender \\ and J. Raymond Pearson
}

It is proposed that rheumatoid deformity of the central three metacarpophalangeal joints is caused primarily by the flexor tendons acting on diseased joint restraints. During pinch and grasp the tendons bend volarly and ulnarly at the tunnel mouth; the resultant pulley forces damage the supporting collateral ligaments, especially on the radial side. The flexor tendons and proximal phalanges can then displace volarly and ulnarly, and the fingers deviate ulnarly. In the fifth digit, ulnar deviation apparently results from the incompletely opposed pull of the abductor digiti quinti.
Es postulate que deformitate rheumatoidee del tres central articulationes metacarpophalangee es causate primarimente per le tendines flexori in lor action contra le resistentia de morbide articulationes. In le activitate del pincear e del saisir, le tendines se flecte in direction volar e ulnar al bucca del tunnel. Le resultante fortias compromitte le supportante ligamentos collateral, specialmente al latere del radio. Alora le tendines flexori e le phalanges proximal pote displaciar se in direction volar e ulnar, e le digitos suffre un deviation in direction ulnar. In le quinte digito, deviation in direction ulnar resulta apparentemente ab le incompletemente opponite traction del quinte abductor digital.

W HEN LOOKING for the major cause of deformity in the rheumatoid metacarpophalangeal joint, several clinical observations offer clues. The characteristics of the early deformity recur with remarkable consistency, being comprised of varying degrees of luxation of the proximal phalanx in a volar and an ulnar direction, and deviation in an ulnar direction. ${ }^{1-4}$ Secondly, the deformity has both passive and dynamic aspects. Passively, the finger can be luxated and deviated more than normal, even though it may be readily returned to a normal position in many patients. ${ }^{5,6}$ Dynamically, the finger tracks along a more luxated and ulnarly deviated path during voluntary active flexion. ${ }^{7}$

The passive deformity implies a loss of integrity of the specific structures normally preventing deviation and luxation. This damage in turn is indicative of forces acting consistently in the direction of the passive deformity.

The dynamic deformity implies a new balance of forces during MCP flexion, which are in dynamic equilibrium only when the finger is tracking along a more ulnar and luxated path. In fact, some patients are totally unable to

Read in part at the Eighteenth Annual Meeting of the American Society for Surgery of the Hand. January 18, 1963, Miami Beach, Florida.

This study was supported in part by grants from the Vocational Rehabilitation Administration, Department of Health, Education and Welfare; and the Michigan Sister Kenny Foundation. 
move the finger actively along a normal path even though they can do it passively with ease. Thus, the disease must in some way consistently alter the equilibrium of forces in the direction of the dynamic deformity.

When considering these two aspects of the deformity, a question arises as to which comes first. If the deformity is initially passive, the potentially causative forces must be present in the healthy hand, but not productive of deformity until the joint restraints are diseased and unable to balance these destructive forces. If the deformity is initially dynamic, the force generators themselves, e.g. the muscles acting on the fingers, must be so altered by the disease that they act consistently in a direction of deformity even before the joint architecture is disturbed. Clinical evidence for the latter is limited.

There seems to be more promise in the supposition that initial deformity is passive. If correct, it means that the potentially disabling forces in the healthy hand should be identifiable, and that the joint restraints normally balancing these forces should consistently show damage in the deformed hand. It also means that damage to these restraints should lead in turn to the dynamic aspect of the deformity. The purpose of this paper is to explore these possibilities as a test of the hypothesis.

\section{Analysis of Forces Operative During Pinch}

In the search for potentially deforming force components, an analysis of the internal forces normally acting on the metacarpophalangeal joint has been made. The functions of pinch and grasp have been selected for this analysis because they constitute much of the forceful activity of the hand ${ }^{8-10}$ The detailed analysis has dealt with tip pinch, but the results are generally applicable to palmar pinch and the various forms of grasp as well.

It can be shown with electromyographic tracings that, during strong pinch or grasp, all muscles crossing the metacarpophalangeal joints of the fingers are consistently active except the long finger extensors. Although methods are not now available for measuring the contraction of each muscle directly, the relative strengths can be estimated analytically by treating finger segments as free bodies in equilibrium. In this way the magnitude of the various muscle forces required by finger geometry to maintain static equilibrium during pinch can be calculated.

Figure 1 shows portions of an index finger beyond the distal and proximal interphalangeal and metacarpophalangeal joints respectively, together with vectors representing the muscle forces having moments about the joint flexion-extension axes during tip pinch in a typical hand. (The compressive forces acting at the joints have zero moments about the axes, and are therefore not shown.) Equilibrium requires that the sum of all moments acting about the axes be zero. Thus, three moment equations are available, one for the flexion-extension axis at each joint. These can be solved simultaneously to yield the magnitudes of three muscle forces relative to the pinch force, $F$. The three selected were the forces produced by the flexor digitorum profundus ( $P$ ), the Hexor digitorum sublimis $(S)$, and the average of the inter- 


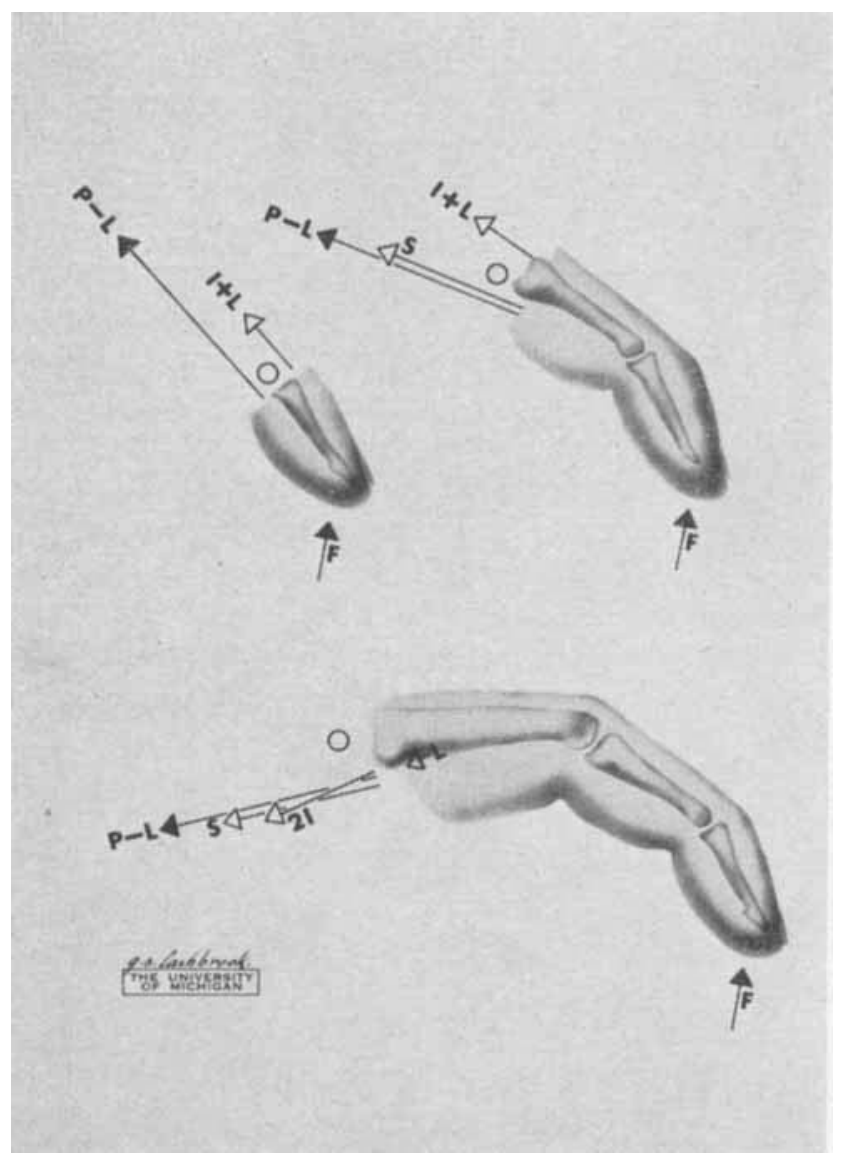

Fig. 1.

ossei (I). To reduce the actual system of muscle forces to these three, the following simplifying assumptions were made.

1. The interossei on the radial and ulnar side have approximately equal moment arms about the flexion-extension axes of the metacarpophalangeal joints, so that the sum of the interosseous forces can be treated as a single force, 21, (even though the two interossei may contribute unequally to the total force). Cadaver measurements have indicated that this assumption is justified.

2. Only half of the total interosseous force reaches the insertion beyond the proximal interphalangeal joint; therefore interosseous forces of $I$ act at both the proximal and distal interphalangeal joints.

3. The lumbrical (L), as it is much smaller than the interosseous, exerts a force of only 1/3I. Although this and the preceding assumption are arbitrary, they do not appreciably affect the conclusions as will be shown.

The three moment equations were solved, using dimensions obtained from the index finger of a cadaver hand in' a position of tip pinch. The equations 
yielded values of $\mathrm{P}=3.8 \mathrm{~F}, \mathrm{~S}=2.5 \mathrm{~F}, \mathrm{I}=0.9 \mathrm{~F}, \mathrm{~L}=0.3 \mathrm{~F}$. The results indicate that during tip pinch the flexor tendons carry large forces: a net value $(\mathrm{S}+\mathrm{P}-\mathrm{L})$ of $6 \mathrm{~F}$, compared to intrinsic muscle forces having a net value $(2 \mathrm{I}+\mathrm{L})$ of only 2.1F. As for assumptions 2 and 3, calculated flexor tendon forces varied no more than' \pm 5 per cent when the lumbrical force was assumed to fluctuate between 0 and 100 per cent of the average interosseous force, or when the portion of the interosseous force reaching the distal interphalangeal joint was assumed to range between 0 and 100 per cent.

These values, of course, pertain only to a single finger in a single position. Values would vary from finger to finger and from hand to hand, depending on details of hand geometry and finger contour during pinch. Such variations, however, would not alter the fact that the flexor tendon forces are many times larger than the intrinsic muscle forces and the pinch force.

For equilibrium to be established there must also be counteracting bonecompressive forces. When calculated by use of a force polygon (fig. 2), it can be shown that these compressive forces become progressively larger at each more proximal joint. In the finger pictured in figure 2, the head of the metacarpal exerts a force of $7.5 \mathrm{~F}$ against the base of the phalanx, or 22.5 pounds during a 3 pound pinch.

\section{Passive Deformity at the Metacarpophalangeal Joint}

If it is assumed that the passive form of metacarpophalangeal joint deformity results from normally present muscle forces acting on diseased joint restraints, the causative forces should be identifiable by finding which restraining structures normally prevent deformity, and which muscle forces act on these restraints in the healthy hand. It can be shown in the cadaver that the only ligamentous structures necessary to prevent passive volar and ulnar subluxation and abniormal ulnar deviation are the more distal fibers of the collateral ligaments (the metacarpophalangeal ligaments). In fact, these are the fibers which define the normal limits of joint motion, and if they and the joint surfaces are intact the finger cannot be placed passively in a position of deformity, even though the extensor mechanism and dorsal capsule are completely removed.

However, if the metacarpophalangeal ligaments are stretched, and the radial side is elongated more than the ulnar, the finger can be luxated passively in both a volar and ulnar direction, and deviated abnormally in an ulnar direction. That such elongation or tearing of collateral ligaments does occur in the rheumatoid hand has been reported by many authors. ${ }^{5,11-15}$ Although the way that rheumatoid ligaments might differ from normal ligaments in such properties as tensile strength, plasticity, and viscoelastic characteristics is poorly understood, the fact that the deformities do occur is evidence in itself that the ligaments are altered and are more susceptible to deformity-producing forces. It is also possible, of course, that some of the slackness observed in the ligaments could be the result of selective destruction' or compression of the joint surfaces, ${ }^{15}$ but even though this occurs, 


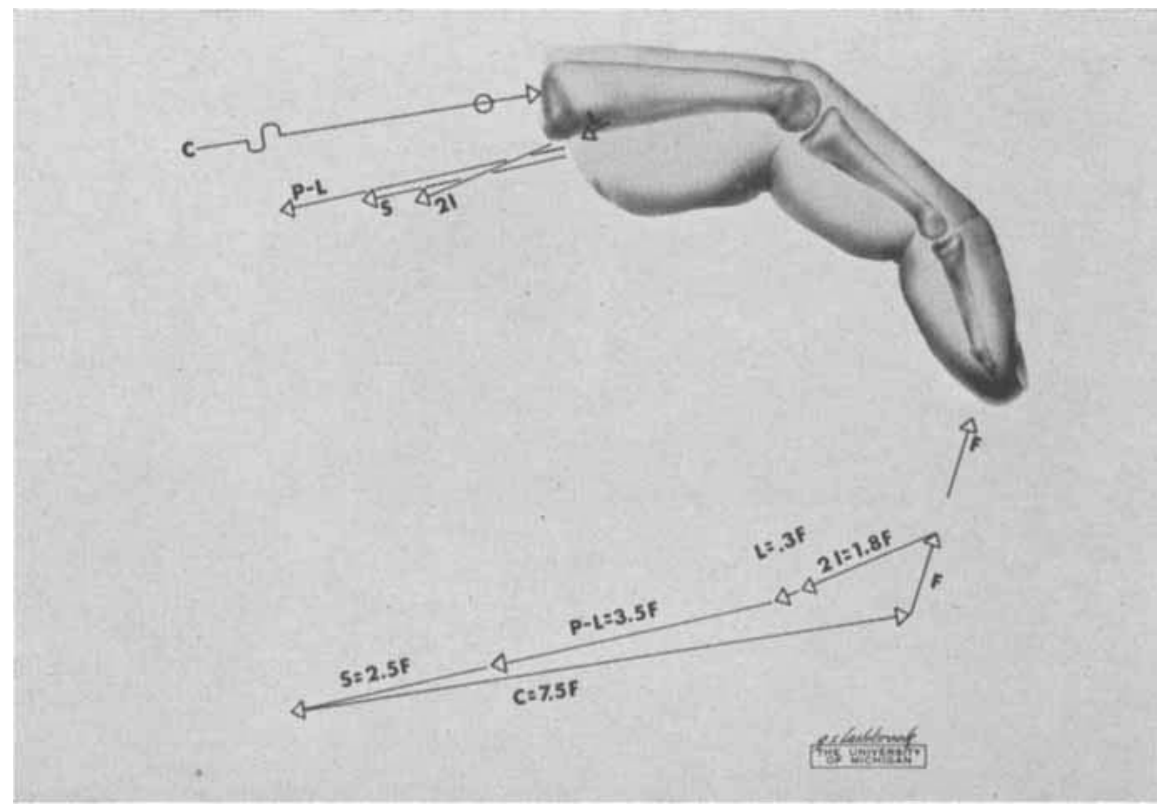

Fig. 2.

the degree of deformity observed usually exceeds that expected from boney and cartilaginous changes alone.

Volar Subluxation: The interosei ${ }^{2,5,12,13}$ and the long flexors ${ }^{5,16}$ have both been incriminated as causing volar subluxation. Because of their direction of pull, the interossei normally exert a volar displacement force that increases as metacarpophalangeal flexion progresses. It is presumed that this deforming action is greater when contractures develop in these muscles and hold the joint in flexion and luxation.

As was shown in the force analysis, however, the tension generated by the interossei during pinch or grasp is relatively small compared to that of the flexors. At the same time, inspection of the anatomy of the metacarpophalangeal joint reveals a way that these large flexor forces can ultimately be transmitted to the metacarpophalangeal ligaments. As the flexor tendons enter the flexor tunnel during pinch they make a sharp volar bend, causing the tunnel mouth and proximal tunnel fibers to act as a pulley. As described by Landsmeer, ${ }^{17}$ the pulley is anchored by the attachments of the tunnel to the volar accessory ligament, which in turn is supported on' either side by the proximal fibers of the collateral ligaments (the metacarpoglenoidal ligaments ${ }^{7}$ ) fanning out from the metacarpal tubercles (fig. 3). This sling arrangement, which is not generally appreciated, holds the tunnel mouth in position without restricting the excursion of flexion-extension. In' cadaver hands, volar pull on the proximal tunnel fibers makes the metacarpoglenoidal ligaments taut, and when these ligaments are cut, the anchorage of the mouth is largely lost. Removal of the extensor mechanism does not compro- 


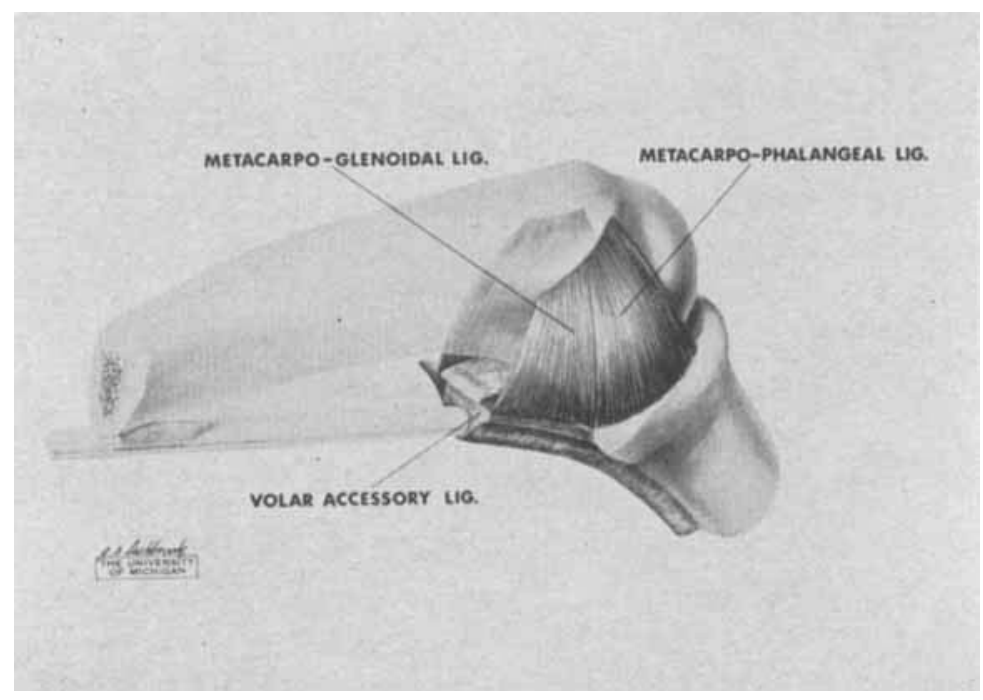

Fig. 3.

mise the function of this sling arrangement. With the type of pinch illustrated in fig. 4, flexor tendon forces of $6 \mathrm{~F}$ would result in a volar pulley force of about $3 \mathrm{~F}$. Thus, during a pinch of 3 pounds the flexors would apply a volar force of approximately 9 pounds to the proximal tunnel and its anchorage system.

If in the presence of the rheumatoid process the metacarpoglenoidal ligaments supporting the tuninel mouth were stretched or torn by the large volar pulley force, the tunnel mouth would be displaced volarly and the load would be transferred to the tunnel's attachment at the proximal phalanx. This would give rise to a new equilibrium of forces acting on the finger. Volar subluxation would now be limited by the dorsal rim of the phalanx's articulating surface, and by the metacarpophalangeal ligaments inserting into the phalanx itself. If these structures were also damaged by the subluxating forces, the base of the phalanx would drop volarward (fig. 5). Flatt has observed that the rim of the joint (and sometimes the head of the metacarpal) does indeed wear down, ${ }^{5}$ and this plus the slackening of the metacarpophalangeal ligaments allows the deformity to progress.

Ulnar Subluxation and Deviation. The large flexor forces in the healthy hand are also capable of causing ulnar deviation and subluxation in digits 2, 3 and 4. During grasp, the flexor tendons in these fingers make an ulnar as well as volar bend at and adjacent to the tunnel entrance (fig. 6). As indicated in figure 7 , the resultant ulnar pulley forces may be as large as $2 \mathrm{~F}$ in the index finger, or 6 pounds during a 3 pound pinch.

The pulley anchor which must balance this added load, consists mainly of the radial metacarpoglenoidal ligament and, in digits 3 and 4 , the deep transverse intermetacarpal ligaments. Stretching or tearing of these structures in the rheumatoid hand would result in displacement of the tunnel 


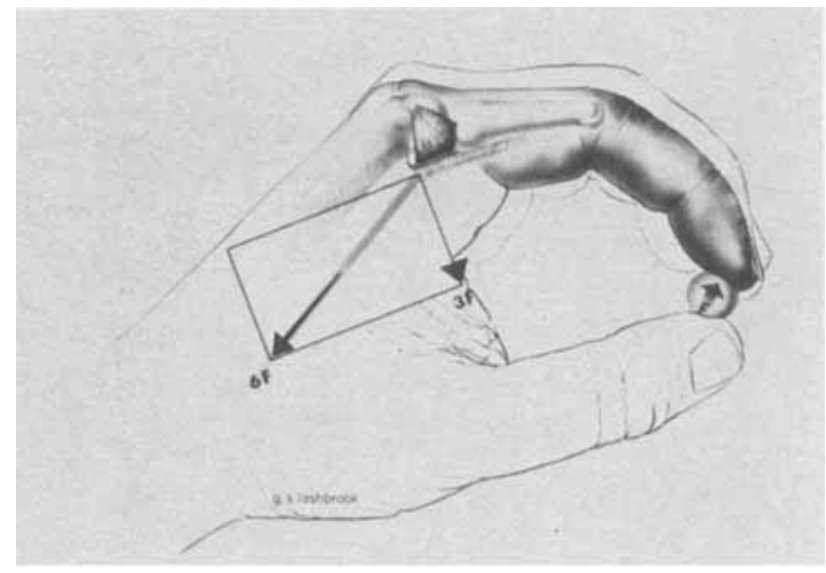

Fig. 4.

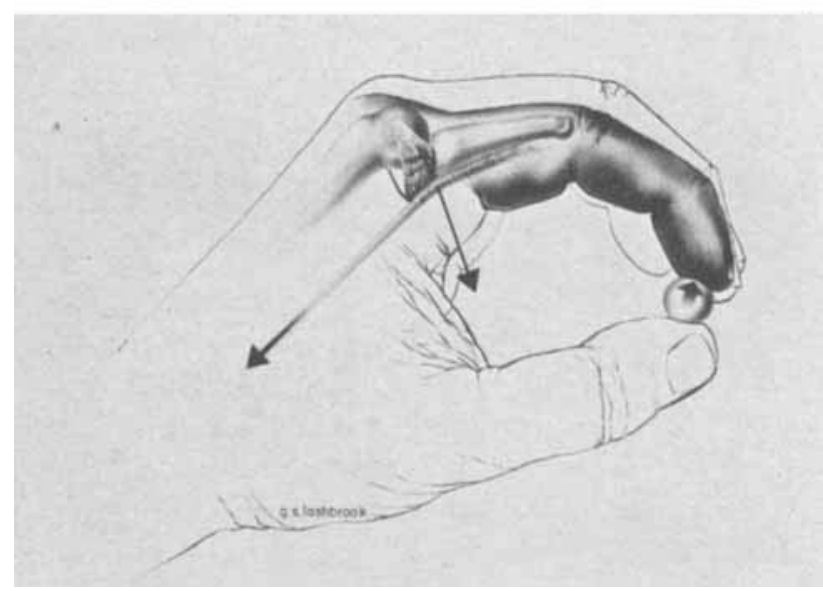

Fig. 5.

mouth ulnarly as well as volarly, and would shift the pulley anchorage to the proximal phalanx and the radial metacarpophalangeal ligament. Loss of integrity of the latter would permit the phalanx to be luxated and deviated abnormally in an ulnar direction.

Figure 8 shows the magnitude of tunnel mouth displacement seen in digits 2,3 , and 4 of a rheumatoid cadaver hand as compared to a normal hand (fig. 6). This shift, along with the associated ulnar subluxation and deviation of the proximal phalanges, is even more apparent on x-ray (fig. 9). In an oblique view of the rheumatoid hand (fig. 10), the volar displacement of the tunnel mouth of the index finger is similarly illustrated. This view also shows destructive changes along the dorsoradial rim of the articulating surfaces, particularly of the fourth and fifth phalanges. These fairly extensive changes in all likelihood developed relatively late and resulted from the large compressive and luxating forces acting on bone weakened by the rheumatoid 


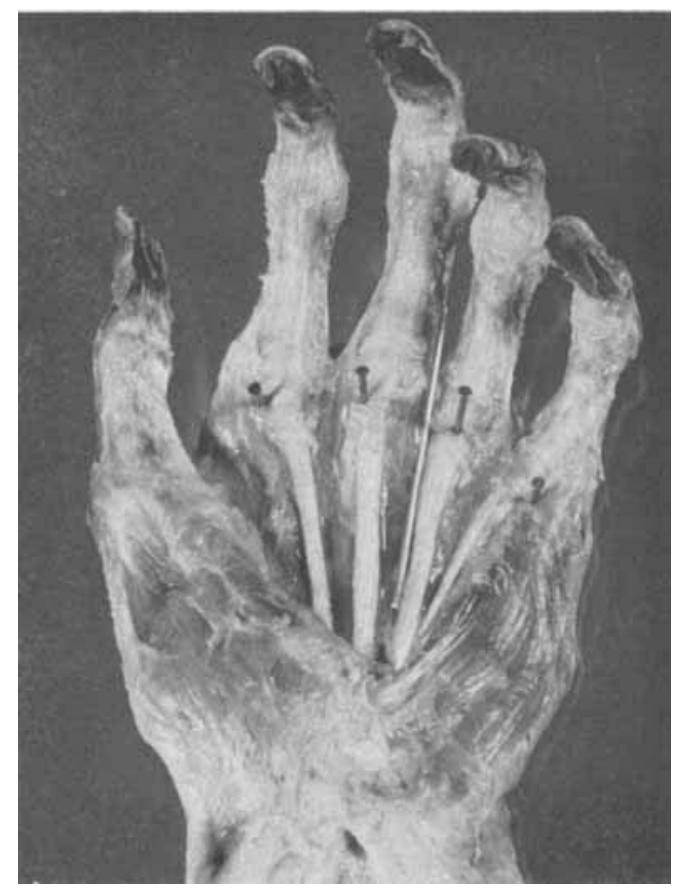

Fig. 6.

process. The importance of compressive forces in production of bone erosion observed radiologically has been pointed out by Martel..$^{18-20}$

Thus, in the normal hand, consistent and sizable forces can be identified which tend to luxate all fingers in a volar direction, and which tend to luxate and deviate digits 2,3 , and 4 in an ulnar direction as well. In each case these forces act on the joint restraints which are ultimately the most damaged in the typical rheumatoid hand, and whose loss results in the passive form of the subluxation and deviation deformities.

\section{Dynamic Deformity at the Metacarpophalangeal Joint}

The hypothesis that passive precedes dynamic deformity must also explain how the dynamic deformity follows. A credible explanation can be based on the alterations which occur in joint geometry as a result of damage to collateral ligaments. Normally, the flexor tendons pass on the ulnar side of the deviation axis in digits 2 and 3 , and over, or close to, the axis in digits 4 and 5 (figs. 6 and 9-a). Evidence for this comes from the direction of deviation of cadaver fingers when the flexor tendons are pulled, and of living fingers when the intrinsic muscles are lost. In the normal hand, the ulnar deviation force of the flexor tendons in the second and third digits is presumably balanced by the radial interossei and lumbricales. If, however, the anchors of the flexor tunnel mouths are damaged and the flexor tendons in digits 2, 3, and 4 bowstring ulnarly, the moment arm of ulnar deviation is increased in digits 2 and 3 , and introduced into the ring finger. Because of the large forces carried by the flexor tendons, even a small ulnar shift at 


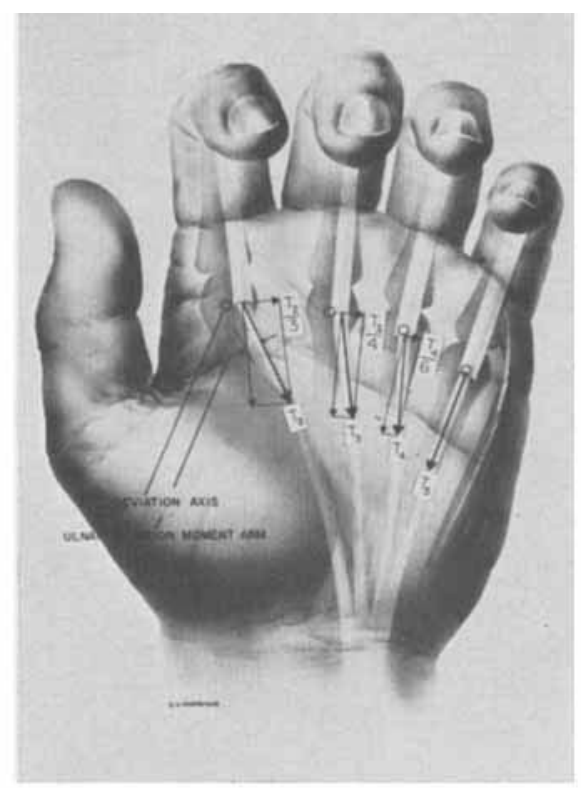

Fig. 7.

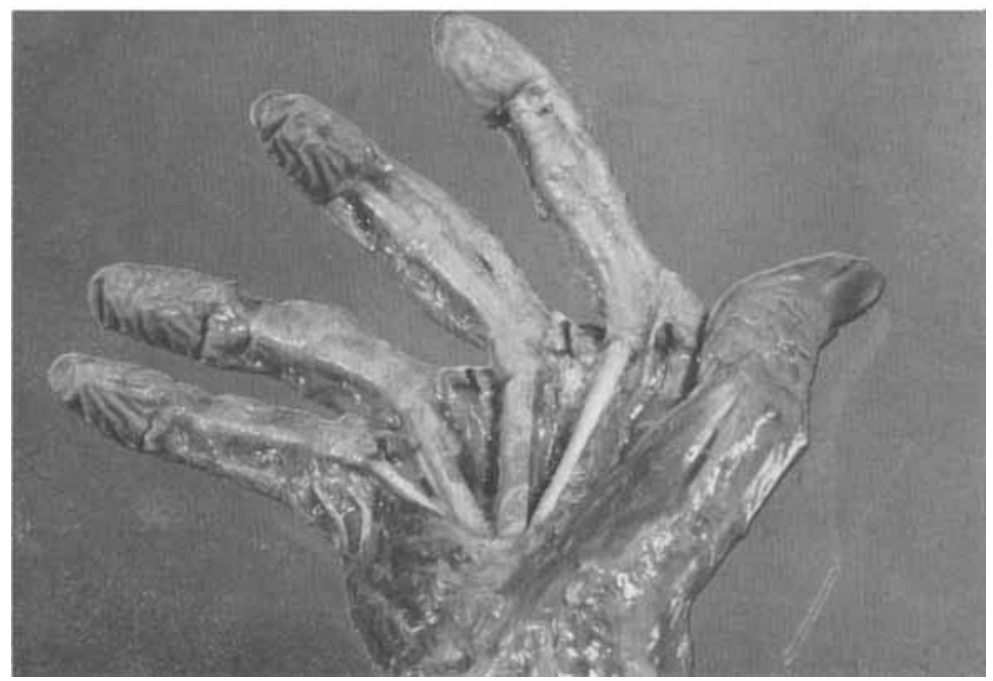

Fig. 8 .

the tunnel mouth would be significant. During a 3 pound pinch by the index finger, a shift of only 0.1 inch would add an ulnar deviation torque of nearly 2 inch-pounds if the flexor tendons carried a force of $6 \mathrm{~F}$. As shown in figs. 8 and $9-\mathrm{a}$, the flexor tendons may in reality be dislocated as far ulnarly as the corresponding extensor tendons sometimes are on the dorsum. With careful palpation clinically, this degree of flexor displacement can be detected in patients with the dynamic deformity as they flex their fingers against resistance. In the rheumatoid cadaver hand depicted, tension on the dis- 


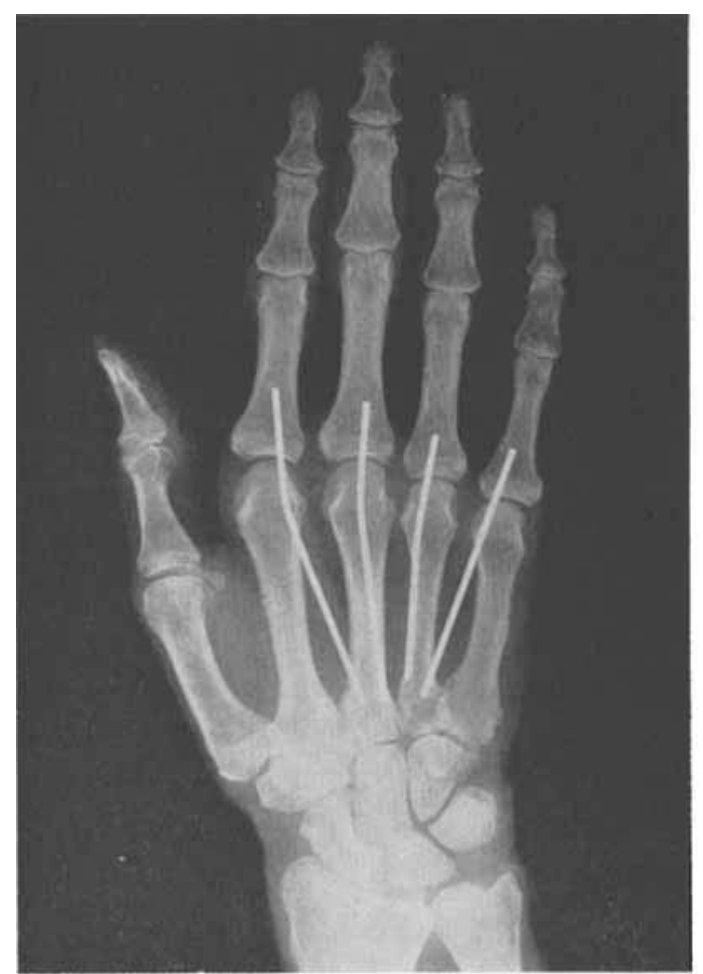

Fig. 9a.

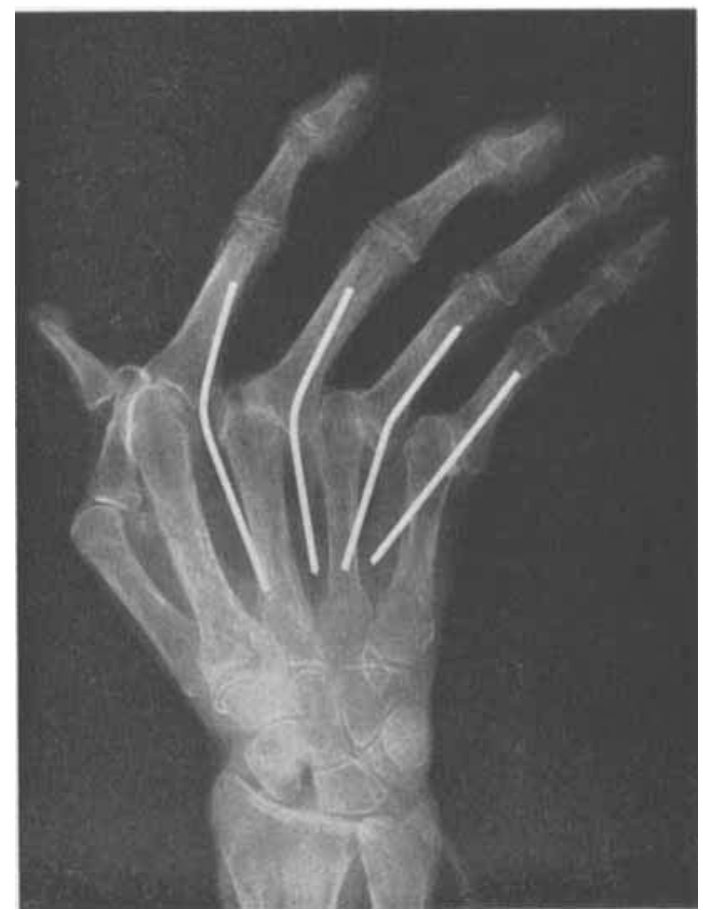

Fig. 9b. 


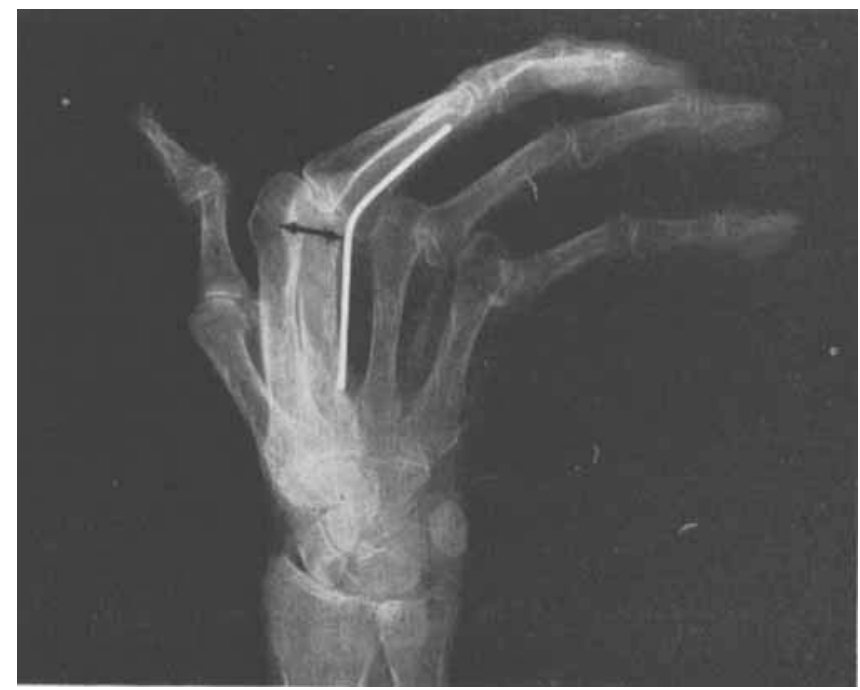

Fig. 10.

placed flexor tendons caused digits 2,3, and 4 to flex along a path of marked ulnar deviation, restrained only by the elongated metacarpophalangeal ligaments on the radial side.

Unless the radial deviators of the fingers can match this increased ulnar deviation torque of the flexors, a new dynamic equilibrium along a more ulnar path will be established during flexion. In many instances, however, the radial deviators are actually less effective than normal. In addition to being weakened directly by the disease process, their mechanical advantages may be reduced. When the proximal phalanx is subluxated volarly and ulnarly, the insertions of the radial interossei are displaced correspondingly, and their moment arms of radial deviation are reduced. The extent of loss of effectiveness can be appreciated clinically by relocating the fingers and observing the apparent increase in strength of contraction that follows.

\section{Deformity at the Fifth Metacarpophalangeal Joint}

In the little finger, the foregoing explanations should hold for the cause of volar subluxation, but not for ulnar subluxation and deviation. Although the flexor tendons bend volarly at the tunnel mouth, they do not bend ulnarly (fig. 6). Anatomically, the fifth digit is in many ways like the thumb, so that the causes and characteristics of deformity in the little finger should reasonably be different just as they are in the thumb. The deformity itself is not only one of subluxation and deviation, but also of outward rotation into a position of opposition. Straub ${ }^{4}$ has proposed that the cause of the deviation deformity in the fifth digit is the incompletely opposed pull of the abductor digiti quinti. This view appears to be supported by anatomic evidence, and is consistent with the concept that the potentially deforming forces are present in the healthy hand.

The hypothenar muscles attached to the ulnar side of the proximal phalanx have more combined mass, and presumably greater strength of contraction, 
than do the volar interosseous and lumbrical attached to the radial side. At the same time, measurements of normal cadaver hands show that the moment arms of the hypothenar muscles producing ulnar deviation and outward rotation, are approximately equal to the offsetting moment arms of muscles on the radial side. Thus, during strong contraction of the hypothenar muscles, some of the counter forces required to maintain equilibrium may be supplied by joint ligaments.

It can be shown on the normal cadaver that if the little finger is rotated outward and abducted slightly, as by the action of the abductor digiti quinti, the fibers of the collateral ligaments are stressed more on the radial than on the ulnar side. That this happens during normal grasp is suggested by the fact that the abductor digiti quinti contracts as the little finger flexes against resistance, and when flexion exceeds about $40^{\circ}$ to $50^{\circ}$ the proximal phalanx is in a position of near-maximum rotation and abduction. Thus the radial collateral ligament may balance some of the forces generated by the abductor digiti quinti during flexion. If this ligament were damaged in the presence of the rheumatoid process, the finger would be displaced and deviated ulnarly and would track along a more ulnar and outwardly rotated path.

\section{Other Potentially Deforming Factors}

Over the years, a multiplicity of causes has been advanced for the ulnar deviation deformity: joint shape, gravity, unilateral capsular damage by the disease process, hand usage, ulnar dislocation of the extensor tendons, and the action of the hypothenar muscles transmitted to the other digits through the deep intermetacarpal ligaments and volar plates. ${ }^{4 \cdot 6}$ Each of these could represent a contributing cause, but for various reasons it is likely that none is the major cause.

As has been' pointed out by Flatt, ${ }^{5}$ neither joint shape, gravity, nor the disease process can be blamed alone, since none act consistently in the direction of deformity. Although it is probably true that much of the forceful usage of the hand tends to deviate the fingers ulnarly, such usage does not explain deformity that occurs before the radial interaossei are too weak to protect against external loads. Similarly, ulnar dislocation of the extensor tendons, although undoubtedly a strong contributing factor, is absent frequently enough to exclude it as a major cause. ${ }^{7,14,21}$ Finally, the fact that deformity may occur in any or all digits except the little finger, argues against the hypothenar muscles serving as more than a contributing cause of deformity in these other digits.

From this analysis it would appear that the other soft-tissue changes commonly seen, such as periarticular scarring and intrinsic muscle contractures, are the result rather than the cause of the initial deformity. Once present, however, they could accentuate or fixate the deformity.,13

\section{Implications}

If it is accepted that the basic metacarpophalangeal joint deformity results from forces generated by the finger flexors and, in the fifth digit, the hypothenar muscles as well, several implications for treatment follow. For de- 
formity prevention, the diseased supports of the flexor tunnel mouths should be protected from the damaging forces exerted by bends in the flexor tendons, and, in the fifth digit, also from the pull of the abductor digiti quinti. This possibly could be accomplished by instructing the patient with acute disease to avoid bending the metacarpophalangeal joints, and to flex only with the interphalangeal joints. A more positive control would be to splint the metacarpophalangeal joints in a position of extension and no deviation. The old exercise of squeezing a rubber ball can only be condemned.

When the deformity has already occurred, the flexor tendons should be relocated to their correct position relative to the axes of deviation and flexionextension. The most fruitful approach to this will no doubt be surgical. Current arthroplasties of the metacarpophalangeal joint are not concerned with the flexor tendons, and the long-term results have often' been disappointing. ${ }^{1}$ It would appear that in addition to the standard procedures, a technique is needed for restoring the integrity of not only the metacarpophalangeal ligaments but also the metacarpoglenoidal ligaments. If such an approach were successful, it would be expected that joint stability would be reestablished and that the flexor tendons would be held in their proper location.

\section{Summary}

It is postulated that the deformities of volar and ulnar subluxation and ulnar deviation in the rheumatoid metacarpophalangeal joint are the result of normally present forces acting on' diseased joint restraints. Specifically, it is proposed that the large volar forces generated by the flexor tendons at the mouth of the flexor tunnel during pinch or grasp cause mechanical damage to the collateral ligaments which serve as anchors. The deformity of volar subluxation results. In digits 2,3 , and 4, additional damage occurs to the radial collateral ligaments as a result of flexor pull on the tunnel mouth in an ulnar direction as well. The resultant ulnar displacement of the flexor tendons causes an ulnar deviation torque and produces the dynamic ulnar deviation deformity. Similar ulnar dislocation of the extensor tendons, although a contributing factor, is not considered a major cause since joint deformity can occur without it. In the little finger, the radial collateral ligament is apparently subject to additional damage from the incompletely opposed pull of the abductor digiti quinti. Ulnar displacement and flexion along a more ulnar path result.

\section{ACKNOWLEDGMENT}

We are indebted to an anonymous donor for the rheumatoid hand depicted.

\section{REFERENCES}

1. Flatt, A. E.: Restoration of rheumatoid finger-joint function. J. Bone and Joint Surg. 43-A:753-774, 1961.

2. Henderson, E. D. and Lipscomb, P. R.: Surgical treatment of rheumatoid hand. J.A.M.A. 175:431-436, 1961.

3. Kuhns, J. G. and Potter, T. A.: Cor- rection of arthritic deformities. In Hollander, J. L.: Arthritis and Allied Conditions. Philadelphia, Lea and Febiger, 1960, pp. 436-467.

4. Straub, L. R.: Surgical rehabilitation of the hand and upper extremity in rheumatoid arthritis. Bull. Rheumat. 
Dis. 12:265-268, 1962.

5. Flatt, A. E.: The Care of the Rheumatoid Hand. Saint Louis, C. V. Mosby Company, 1963, pp. 53-54, 147-155.

6. Lush, B.: Ulnar deviation of the fingers. Ann. Rheum. Dis, 11:219-221, 1952.

7. Brewerton, D. A.: Hand deformity in rheumatoid disease. Ann. Rheum. Dis. 16:183-197, 1957.

8. Landsmeer, J. M. F.: Power grip and precision handling. Ann. Rheum. Dis. 21:164-170, 1962.

9. Taylor, C. L. and Schwarz, R. J.: The anatomy and mechanics of the human hand. Artificial Limbs, 2:22-35, 1955.

10. Napier, J. R.: The prehensile movements of the human hand. J. Bone and Joint Surg. 30-B:902-913, 1956.

11. Fearnley, G. R.: Ulnar deviation of the fingers. Ann. Rheum. Dis. 10:126136,1951 .

12. Steindler, A.: Arthritic deformities of the wrist and fingers. J. Bone and Joint Surg. 33:849-862, 1951.

13. Bunnell, S.: Surgery of the rheumatic hand. J. Bone and Joint Surg. 37-A: 759-766, 1955.
14. Vainio, K. and Oka, M.: Ulnar deviation of the fingers. Ann. Rheum. Dis. 12:122-124, 1953.

15. Brooks, A. L.: The rheumatoid hand problem. Amer. J. Orthoped. 5:168$175,1963$.

16. Rose, D. L. and Wallace, L. I.: A remedial occupational therapy program for the residuals of rheumatoid arthritis of the hand. Amer. J. Phys. Med. 31:5-13, 1952.

17. Landsmeer, J. M. F.: Anatomical and functional investigations on the articulations of the human fingers. Acta Anat. Supp. 24, 25:1-69, 1955.

18. Martel, W., Holt, J. F. and Cassidy, J. T.: Roentgenologic manifestations of juvenile rheumatoid arthritis. Am. J. Roentgenol. 88:400, 1962.

19. - - The pattern of rheumatoid arthritis in the hand and wrist. To be published: Rad. Clin. N.A., 1964.

20. - : Personal communication.

21. Swanson, A. B.: The need for early treatment of the rheumatoid hand. J. Mich. State Med. Soc. 60:348-351, 1961.

Edwin M. Smith, M.D., Associate Professor of Physical Medicine and Rehabilitation, University of Michigan, Ann Arbor, Michigan.

Robert C. Juvinall, M.S.M.E., Professor of Mechanical Engineering, University of Michigan, Ann Arbor, Michigan.

Leonard F. Bender, M.D., Associate Professor of Physical Medicine and Rehabilitation, University of Michigan, Ann Arbor, Michigan.

J. Raymond Pearson, M.S.M.E., Professor of Mechanical Engineering, University of Michigan, Ann Arbor, Michigan. 